remark "He's still a bit tight, doctor" is passed, how quickly a "shot" of "tubarine" secures the desired result, while the reply "He's a very resistant type" satisfies honour; but none marvels at the miracle of what the human body can withstand. Several hours later the patient is just sufficiently conscious to be told that his operation is over, and he sinks once more into the twilight of that half-world known as "recovering from the anaesthetic." $\mathrm{He}$ does recover and reaches convalescence, often without complications; yet none wonders at the miracle. Possibly the picture is overdrawn, but if it is a caricature the implications are nevertheless true.

We use to-day powerful agents which have revolutionized anaesthesia : is it strange therefore to affirm that when they are used the whole conception of anaesthetic administration must also be revolutionized ? Every phase of modern technique affects the respiratory act either centrally or mechanically. The anaesthetist must breathe for the patient, and the old conception that as long as the patient was breathing everything was all right must be forgotten and a new approach substituted. The moment these drugs and these methods are used the anaesthetist is wholly responsible for the gaseous exchange in the lungs of his patient and hap what hap there must be no anoxia. This can only be assured by the constant and intelligent manipulation of the rebreathing bag of a sound closed circuit. Till this is realized, shock, ileus, post-operative vomiting, and many other complications will always occur.

The day of heavy premedication has passed, for with the advent of the intravenous barbiturates the terror of induction has been removed and the slow recovery of consciousness and the essential reflexes, consequent on the production of preoperative peace, should now never be seen. Minimal premedication given at the right time is all that is required nowadays.

Crile's theory of shock is now outmoded; it has been replaced by the solid biological fact that so long as blood loss is made good and provided that there is no anoxia, with few exceptions, the lighter the plane of anaesthesia the more readily can the neurovascular mechanism of the body withstand the onset of shock. It is sufficient, therefore, to maintain the patient in a light first-plane anaesthesia by the judicious use of the intravenous barbiturates, supplemented when necessary by the exhibition of a minimal quantity of cyclopropane, while relixation is secured by the addition of $d$-tubocurarine chloride. When these three drugs are used in combination they potentiate each other in a remarkable way, consequently toxic after-effects are greatly minimized : but there must be no anoxia.

Anaesthesia conducted on these lines, no matter how severe the operation, will present a patient who coughs on the table and will often speak; one who on being returned to the ward is able to sit up immediately and is fully co-operative within half an hour. After this if he is nursed in a steam tent and an adequate fluid intake is assured-by the intravenous route if necessary-the bronchial secretions are kept fluid, with the result that coughing, with the wound area adequately supported, is productive and post-operative complications are avoided.

These few observations result from the experience in the use of $d$-tubocurarine chloride over a period of two and a half years.-I am, etc.,

Liverpool.

JoHn Halton.

\section{Anaesthesia for Caesarean Section}

SIR,-The recent discussion on anaesthesia for caesarean section at the Royal Society of Medicine, reported in your issue of April 5 (p. 463), gives the impression of undiluted and almost unchallenged spinal, epidural, or caudal anaesthesia as being the method of choice. If this is so I feel that it is unfortunate and should not be left uncontested. The various speakers pointed out the disadvantages, such as the need for taking readings of blood pressure during operation, the not infrequent necessity for stimulants, and the fact that it was not suitable for certain types of case, but insufficient stress was laid upon these disadvantages, and the effect upon the patient was barely mentioned.

It must be admitted that an experienced anaesthetist can make a success, surgically speaking, of practically any type of anaesthesia one likes to mention. There is no onus on an obstetrician to make himself familiar with spinal anaesthesia in its various techniques, but there is, I would suggest, every onus upon him to familiarize himself with the details of local anaesthesia. This was referred to by Mr. C. Macintosh Marshall and Prof. Chassar Moir, but it was not sufficiently emphasized that this can often be a life-saving method. It is applicable either alone or with the addition of inhalation or intravenous anaesthesia for any and every case in which this operation is required, and in those in which the patient's condition gives rise to anxiety it is very often the only type of anaesthetic which should be used. If this were more widely recognized a considerable saving of life could undoubtedly be effected.

Its disadvantages lie only in a little added discomfort to the mother and the distaste many of us have for operating upon a conscious patient. The former can be largely avoided by careful handling and good technique, the latter by the use of inhalation anaesthesia in addition, which is, I believe, the real method of choice. It is, however, very hard to better the results obtained from the point of view of both patient and surgeon by a sequence of gas, oxygen, and chloroform, followed, after delivery of the child, by a little ether or what you will given by a competent anaesthetist.-I am, etc.,

London, W.1.

F. NeON Reynolds.

SIR,-The recent interest in this subject at the Royal Society of Medicine (April 5, p. 463) and Dr. T. C. Gray's article on the use of $d$-tubocurarine in caesarean section (April 5, p. 444) will no doubt result in many conflicting opinions. While it is encouraging to read of dissatisfaction with the older methods, it is disappointing to see so little support for the use of local anaesthesia. The only objection appears to be that it is unpleasant for the patient, and even so great an advocate as Mr. C. Macintosh Marshall refers to ameliorating the rigours of this method. In my view the unpleasantness is greatly exaggerated, chiefly by those who have used the method only a few times and who have discarded it after the initial disappointments which inevitably precede the attainment of a satisfactory technique.

Failure is usually due to one of two reasons. The first mistake is to expect too much from local anaesthesia alone. In the lower-segment operation it is practically impossible to deliver painlessly a head deeply engaged in the pelvis without the aid of some additional anaesthetic. Closing the abdomen may also be painful if the operation has not been completed in reasonable time and the effect of the local anaesthetic is wearing off. Both of these disadvantages may be met by the addition of gas and oxygen when necessary. The amount required is minimal, and a high proportion of oxygen can be given, because adequate relaxation should be obtained from the local anaesthetic. The second mistake concerns the actual technique. A considerable amount of deep "fanning out" of the injection is necessary in the suprapubic region. This is where most of the discomfort is felt, and it is aggravated by the pressure of the Doyen retractor. Needless to say the injection is greatly facilitated by the use of a continuous-action syringe. The use of a sucker will minimize the painful swabbing of the peritoneal cavity.

The psychological approach to the patient is also important. Conversation and an explanation of what is happening are a great help, and it should be remembered that most operating tables are extremely hard. The use of "omnopon" and scopolamine immediately after the birth of the child undoubtedly has a retrograde amnesic effect.

In this unit local anaesthesia has been used a great deal, although it was discarded temporarily after the first few attempts for the reasons given above. It is now used more than any other method, but only with the object of delivering the baby entirely with local anaesthesia. After this, although the operation can be completed without further anaesthesia, there is no reason to withhold gas and oxygen, and it is usually given.

If this combined method is planned deliberately, and the addition of gas and oxygen after the birth of the child is regarded not as a "failure of local anaesthesia" but as an essential part of the technique, there should be no need to look for other methods involving complicated apparatus, "aided" respiration, foetal narcosis, and the constant watch for sudden collapse of the mother.-I am, etc.,

North Herts Maternity Unit.

D. W. JAMES. 